\title{
Effect of membrane cholesterol on dynamical properties of solvent molecules
}

\author{
Hadi Rahmaninejad ${ }^{1}$ and Darin D. Vaughan ${ }^{2}$ \\ ${ }^{1}$ Department of Physics, Virginia Tech, Blacksburg, VA 24061 \\ ${ }^{2}$ Sanders-Brown Center on Aging, University of Kentucky, Lexington, KY, United \\ States of America \\ ${ }^{2}$ Department of Pharmacology and Nutritional Science, University of Kentucky, \\ Lexington, KY, United States of America
}

\begin{abstract}
Membrane lipid composition is a critical feature of cell function, where cholesterol is a major lipid sterol component that influences the membranes physical and electrical properties. The effects of cholesterol on transport properties between adjacent to the cells, especially in junctions formed between cells is not completely understood. These junctions where substances transport and signaling is critical may be affected by modifying the cholesterol composition of the membrane in these junctional regions. Here we show how the cholesterol content in a membrane can regulate these phenomena by changing their effect on transport into and through regions between cell membranes in close proximity. Through geometric and electrostatic effects interaction with substrates, the properties of the fluid between membranes are shown to potentially enforce concentration gradients of dissolved compounds that may be biologically significant.
\end{abstract}

\section{Introduction}

Biological membranes are composed of a mosaic of proteins and lipids in a fluid-like matrix, with lipids forming a micelle bilayer with proteins embedded or attached to surface. The precise composition is variable and can range from $20 \%$ protein and $80 \%$ lipid, to $80 \%$ protein and $20 \%$ lipid, depending on the cell and tissue. Proteins are generally divided into two classes; integral (or intrinsic) that pass through the membrane entirely, and peripheral (or extrinsic) proteins that are found on the outer or inner leaflet of a membrane. The membrane bound proteins are believed to be responsible for varieties of functions including cell signaling and interacting with ligands [1]. The possible classes of lipids are of a much greater variety, and the relative composition of each class also depends on the particular organellar membrane, as seen in Figure 1. 
Geometrical properties of the extracellular junctions widely affect gradient in concentration by affecting accessible areas. Furthermore, electrostatic interaction can directly affect substances from the external hydrophilic layer of the membrane. It can regulate it through interaction with water molecules as the primary solvent around cells. Hence, phospholipids as the main constitutes of the membrane plays an essential role, especially by affecting the physical properties of them [2]. Cholesterol molecules, as another key components of membrane, not only regulates mechanical and physical properties of membranes, such as their fluidity, but also modulates the viscoelastic properties of the membrane [3] which can be important for pathogenesis and cellular organization[4]. It had been shown that Cholesterol can increase membrane thickness and area per lipids. they also affect electrostatic interactions by modifying surface charge density of them [5,6]. In particular, increasing Cholesterol can decrease average surface charge density of phospholipid membrane [7]. Cholesterol can alter the surface charge density of membranes as high as $25 \%$ [8], and consequently modify electrostatic interactions.

In this work, we applied molecular dynamic simulations to investigate how the Cholesterol composition rates thickness of the membrane and surface charge density. Then we investigate how these changes regulates dynamical properties of the solvent molecules such as diffusion coefficients near the membranes. Finally we discussed how cumulative effect of those dynamic properties influence mass transport in physiological environments, especially in extracellular junctions.

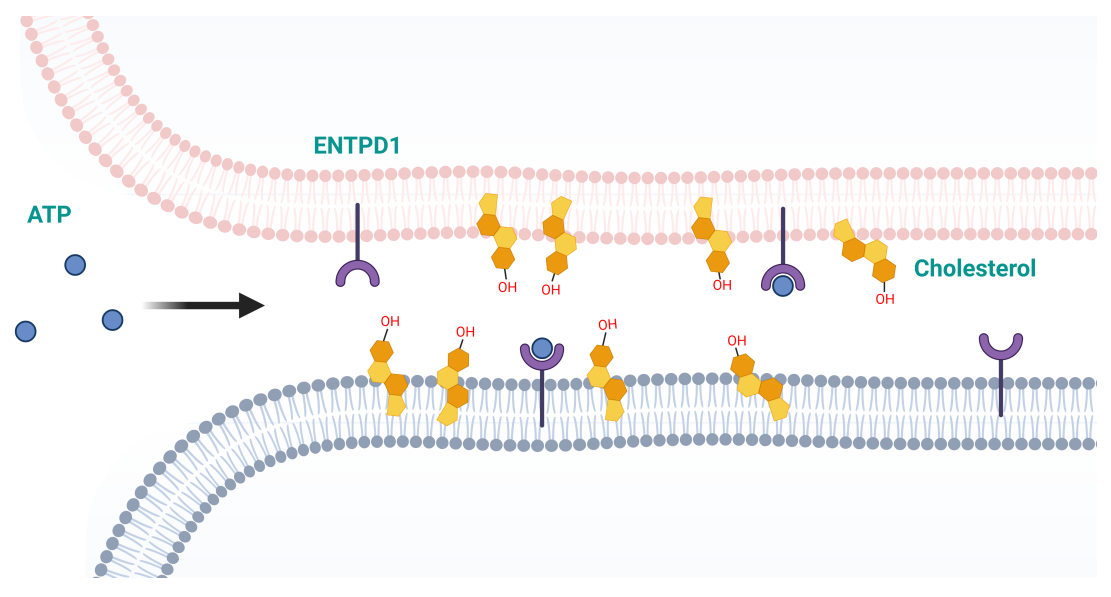

Figure 1: General system design

\section{Result}

To investigate the effects of surface Cholesterol density on the solvent molecules and ions' transport properties, we required to estimate how the rate of Choles- 
terol per area influences the average diffusion coefficient. This sub-goal will help us to construct our hypothesis toward how Cholesterol regulates membranes' functionality in the sense of its absorption properties and then on other features such as mass transport in ion channels.

\section{Effective thickness}

To compute thickness of the membrane, first, we calculated the z-density of water molecules in Fig. 2, which provides a view of membrane boundary for water molecules. In this figure, the $\mathrm{x}$-axis shows the $\mathrm{z}$ coordinate, which is perpendicular to the membrane's surface according to Fig. 5. Blue and red lines show water density in number per length of z-axis for a low and high percentage of Cholesterol, respectively, which contains the total number of water molecules in an $\mathrm{x}-\mathrm{y}$ surface.

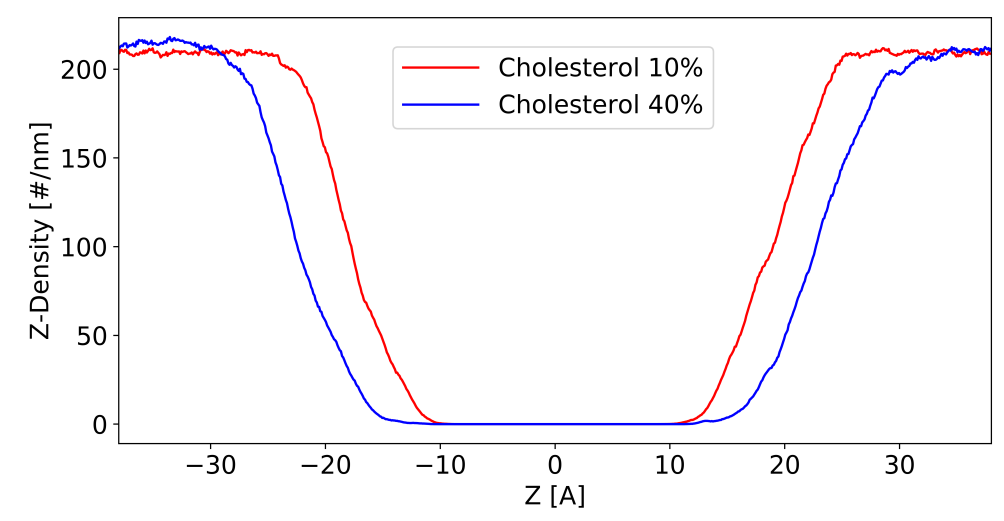

Figure 2: Water density along z axis

We define an effective thickness for lipid membranes as the thickness with no water molecules. In that case, this figure is clearly showing the increase in Cholesterol content increases effective thickness of the lipid membrane. Other computational and experimental works indicate such proportional relation between membrane thickness and Cholesterol content [9, 10], with computing "peak-to-peak" distances using electron density profile.

\section{Surface charge density}

Then we calculated average partial charge along z-axis in Fig. 3. A more negative charge at the membrane boundaries with relatively less amount of Cholesterol indicates higher net surface charge density with decreasing Cholesterol. To better show this, we offer a residue view vs. charged view of DMPC membrane, 
including Cholesterol in Fig. 3. The less sharp color of the area with Cholesterol indicates how it smoothens the membrane's hydrophobic surface.
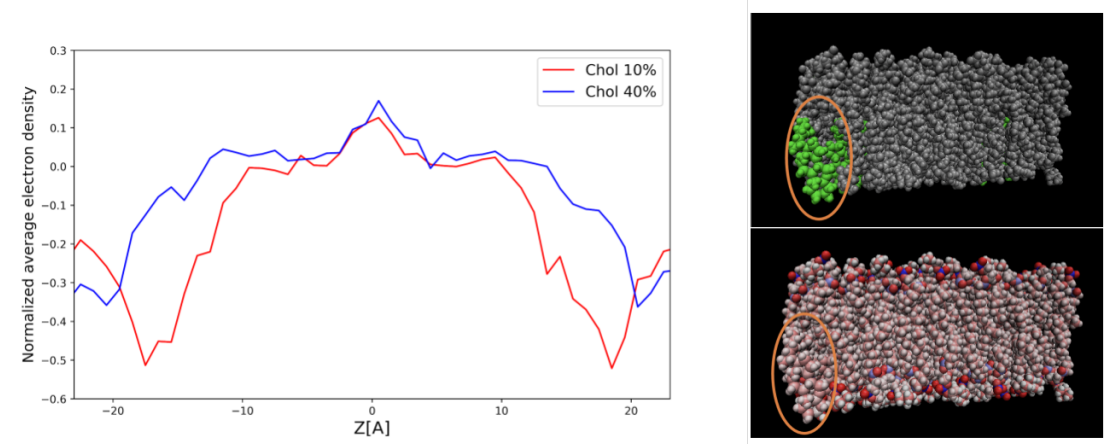

Figure 3: Partial charge of membrane molecules along z-axis. Top right is showing a residue view of the membrane surface with Cholesterol in green, while bottom right is showing the same surface in charge view.

\section{Diffusion coefficient}

To analyze the direct effect of higher charge surface density of membrane with less cholesterol, we computed average diffusion coefficients of water molecules using the Mean Squared Deviation (MSD), both close and far from the membrane. We did this procedure for both cases of high- and a low percentage of cholesterol molecules in Fig. 4.

Far from the membrane, diffusion is almost independent of the Cholesterol content in the membrane, which is equal to the diffusion in the bulk case. However, close to the membrane, diffusion is positively affected by the amount of Cholesterol. More importantly, a higher Cholesterol percentage is increasing diffusivity adjacent to the membrane. 


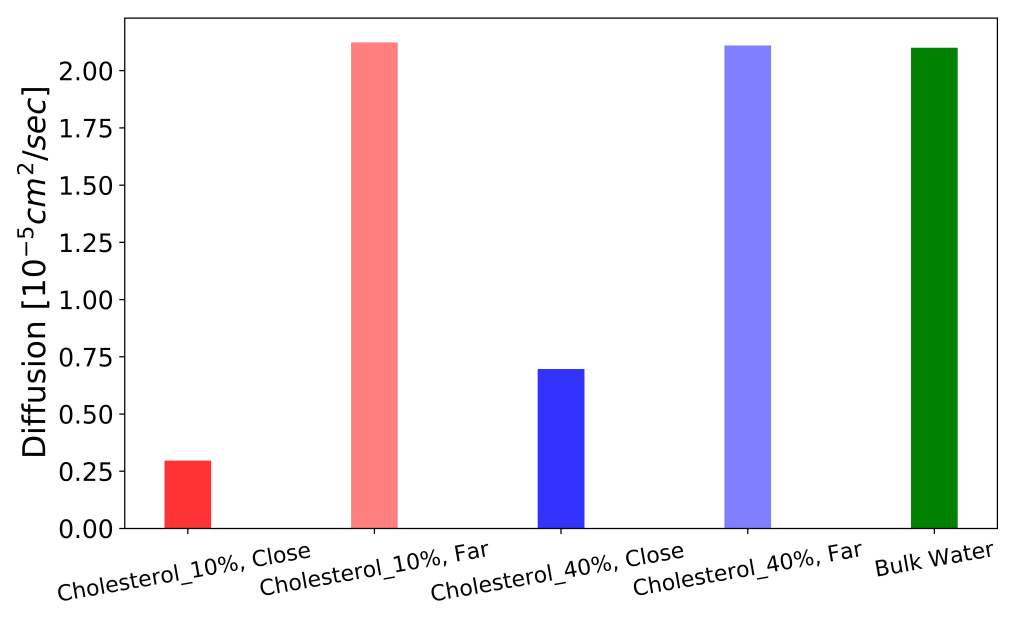

Figure 4: The diffusion coefficient for water molecules close and far from the membranes with different percentages of Cholesterol, in comparison with water diffusion in bulk media

\section{Discussion}

We showed that Cholesterol increases the membrane's thickness and decreases the surface charge density of the membrane. As a result, water molecules are averagely less attracted to a membrane with a higher Cholesterol level. Water molecules are attracted more to the hydrophilic head of the phospholipid molecules than Cholesterols. Having higher electrostatic interaction between phospholipids and water molecules, the dynamical properties such as diffusion coefficient are highly changing in the membrane's vicinity. Fig. 4 shows that the diffusion coefficient substantially drops for water molecules close to the membrane. This phenomenon is more significant for membranes with less Cholesterol, owing to stronger electrostatic interactions in that area. A substantial difference in diffusion coefficient near the surface for membranes with variant amount of Cholesterol is an indication of how electrostatical interaction is changing with Cholesterol. Far from the membrane, as the interaction is less intensive, we anticipate that diffusivity is similar to the bulk case, as shown in Fig. 4, for both less and high Cholesterol content layers. This feature leads to a less receptive layer and is more resistant to water molecules and consequently increases the membrane's thickness without waters, as can be seen in Fig. 2. The electrical interaction is more potent for adenosine substrates in very tight junctions. They are non-zero charged particles and can be influenced favorably by attraction/repulsion forces in that area. A more attractive junction to the substrate increases the substrates' concentration in the intersection relative to 
a less attractive wall. Previous works [11] proved that how the distribution of nucleotides changes within extracellular junctions with confinement and electrostatic potential of the membranes. Hence, Cholesterol content in the membrane is expected to significantly change nucleotides' distributions by changing junction width by influencing the thickness and the membrane's electrical potential. Given recent advances in the medical imaging technologies such as those in the ultra-high-field MRI hardware and software [2, 12, 13], the potential of validating this study in comparison with the diffusion images obtained by the ultra-high signal-to-noise ratio technologies is on the horizon.

\section{Molecular dynamic simulation}

The original structure of the DMPC membrane is constructed using charmGUI/membrane builder. Force field parameters of lipid membrane and Cholesterol are based on the work of MacKerell et al. [14]. Similar structures are also provided by [15] and a database for different types of the lipid membrane. The total number of lipid molecules in each layer of the membrane is 100, so that the percentage indicates the number of cholesterol molecules as well. To create a lipid membrane with a higher number of lipid molecules, like 512 lipid membrane, a $2 \times 2$ replication of the 128 lipids in the XY plane with "gromacs" tool "gmx genconf" can be used. The structure was then solvated in an $83 \mathrm{~A} \mathrm{x}$ $83 \mathrm{~A}$ water box using "packmol," as shown in Fig. 5. It was minimized and equilibrated after solvation and then was run for $3 \mathrm{~ns}$ in $293 \mathrm{~K}$ in an NVT ensemble. To calculate electron density along the z-axis, we used the density profile tool in VMD [16] which allows us to compute 1-D atomic density along an axis.

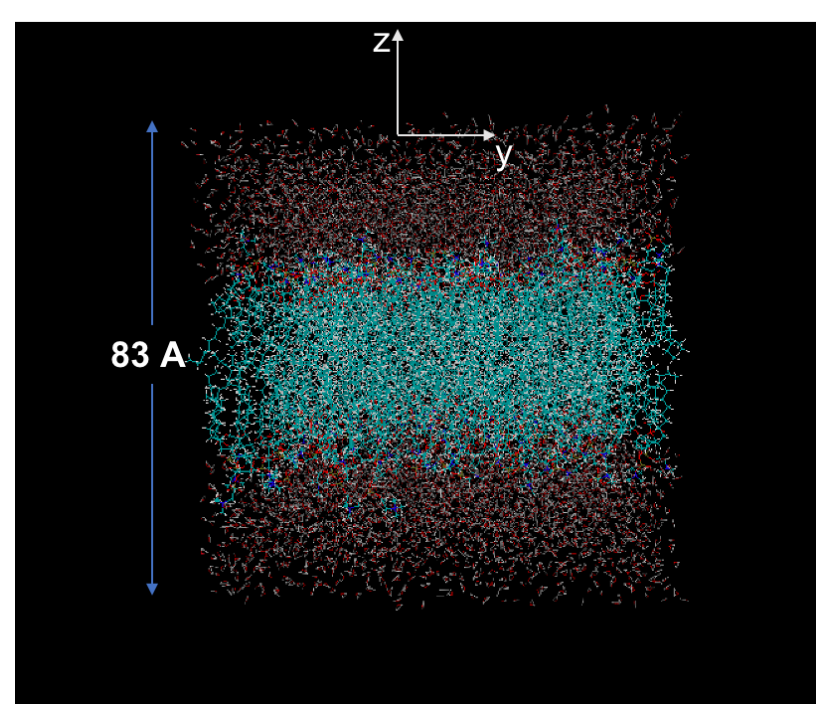

Figure 5: Configuration of the membrane 


\section{References}

[1] K. R. Neupane et al. "Macrophage-Engineered Vesicles for Therapeutic Delivery and Bidirectional Reprogramming of Immune Cell Polarization". In: ACS Omega 6.5 (2021), pp. 3847-3857.

[2] C. Päslack et al. "Hydration-mediated stiffening of collective membrane dynamics by cholesterol". In: Phys. Chem. Chem. Phys. 21 (20 2019), pp. 10370-10376.

[3] Z. Al-Rekabi and S. Contera. "Multifrequency AFM reveals lipid membrane mechanical properties and the effect of cholesterol in modulating viscoelasticity". In: Proceedings of the National Academy of Sciences 115.11 (2018), pp. 2658-2663. ISSN: 0027-8424.

[4] J. N. Sandin et al. "Near Simultaneous Laser Scanning Confocal and Atomic Force Microscopy (Conpokal) on Live Cells". In: Journal of visualized experiments : JoVE 162 (Aug. 2020). ISSN: 1940-087X.

[5] F. de Meyer and B. Smit. "Effect of cholesterol on the structure of a phospholipid bilayer". In: 106.10 (2009), pp. 3654-3658.

[6] E. Bastiaanse, K. M. Höld, and A. Van der Laarse. "The effect of membrane cholesterol content on ion transport processes in plasma membranes". In: Cardiovascular Research 33.2 (Feb. 1997), pp. 272-283. ISSN: 0008-6363.

[7] A. Magarkar et al. "Cholesterol level affects surface charge of lipid membranes in saline solution". In: Scientific Reports 4.1 (2014), p. 5005.

[8] J. Forne's and J. Procopio. "Influence of cholesterol on the surface charge density and surface potential of lipid bilayer membranes". In: Journal of Colloid and Interface Science 117.2 (1987), pp. 570-573. ISSN: 0021-9797.

[9] G. Khelashvili, G. Pabst, and D. Harries. "Cholesterol Orientation and Tilt Modulus in DMPC Bilayers". In: The Journal of Physical Chemistry B 114.22 (2010), pp. 7524-7534.

[10] A. Hodzic et al. "Differential Modulation of Membrane Structure and Fluctuations by Plant Sterols and Cholesterol". In: Biophysical journal 94 (June 2008), pp. 3935-44.

[11] H. Rahmaninejad et al. "Co-localization and confinement of ecto-nucleotidases modulate extracellular adenosine nucleotide distributions". In: PLOS Computational Biology 16.6 (June 2020), pp. 1-31.

[12] N. Tavaf et al. "GRAPPA-GANs for Parallel MRI Reconstruction". In: arXiv preprint arXiv:2101.03135 (Jan. 2021). arXiv: 2101.03135.

[13] N. Tavaf et al. "A Self-Decoupled 32 Channel Receive Array for Human Brain Magnetic Resonance Imaging at 10.5T". In: arxiv preprint arXiv: 2009.07163v2 (Sept. 2020). arXiv: 2009.07163. 
[14] K. Vanommeslaeghe et al. "CHARMM general force field: A force field for drug-like molecules compatible with the CHARMM all-atom additive biological force fields". In: Journal of Computational Chemistry 31.4 (2010), pp. 671-690.

[15] J. P. M. Jämbeck and A. P. Lyubartsev. "Another Piece of the Membrane Puzzle: Extending Slipids Further". In: Journal of Chemical Theory and Computation 9.1 (2013), pp. 774-784.

[16] T. Giorgino. "Computing 1-D atomic densities in macromolecular simulations: The density profile tool for VMD". In: Computer Physics Communications 185.1 (2014), pp. 317-322. ISSN: 0010-4655. 